\title{
$G$-intersecting Families
}

\author{
Tom Bohman Alan Frieze* Miklós Ruszinkótł \\ Luboš Thoma ${ }^{\S}$ \\ Department of Mathematical Sciences, \\ Carnegie Mellon University.
}

October 12, 1999

\begin{abstract}
Let $G$ be a graph on vertex set $[n]$, and for $X \subseteq[n]$ let $N(X)$ be the union of $X$ and it's neighborhood in $G$. A family of sets $\mathcal{F} \subseteq 2^{[n]}$ is $G$-intersecting if $N(X) \cap Y \neq \emptyset$ for all $X, Y \in \mathcal{F}$. In this paper we study the cardinality and structure of the largest $k$-uniform $G$-intersecting families on a fixed graph $G$.
\end{abstract}

\section{Introduction}

An intersecting family is a collection of sets $\mathcal{F} \subseteq 2^{[n]}$ such that

$$
X, Y \in \mathcal{F} \Rightarrow X \cap Y \neq \emptyset \text {. }
$$

The classic theorem of Erdős-Ko-Rado [5] states that a $k$-uniform intersecting family on a ground set of size $n$ contains at most $\left(\begin{array}{l}n-1 \\ k-1\end{array}\right)$ sets. Furthermore, a family achieves this extrema if and only if the family consists of all $k$-sets containing some fixed element of $[n]$. For a general introduction to the theory of intersecting families see $[3]$.

We consider a generalization of the notion of an intersecting family (for a survey of other generalizations of the Erdős-Ko-Rado theorem see [4]).

First consider the following: In an intersecting family $\mathcal{F}$ we require that $X, Y \in \mathcal{F}$ should intersect. Suppose that we relax this to simply require that $X, Y \in \mathcal{F}$ are "close" in some sense. Can something interesting be said? We believe that it can. For example suppose that we take " $X, Y$ are close" to mean that there exist $x \in X, y \in Y$

\footnotetext{
* Supported in part by NSF grant CCR-9530974.

†Permanent Address: Computer and Automation Research Institute of the Hungarian Academy of Sciences, Budapest, P.O.Box 63, Hungary-1518.

${ }^{\ddagger}$ Research was partially supported by OTKA Grants T 030059 and T 29074, FKFP 0607/1999 and by the Bolyai Foundation.

$\S$ Supported in part by NSF grant DMS-9970622.
} 
such that $\operatorname{dist}(x, y)=|x-y| \leq 1$ (it will be convenient to have $\operatorname{dist}(n, 1)=1$ here). What can we say about the maximum size of a $k$-uniform family where every pair of distinct sets are close? A moments thought suggests that a candidate for a maximal $\mathcal{F}$ is the set of $k$-sets which contain 2 or 3 . This is almost correct. Throwing in those sets which contain both 1 and 4 gives the correct answer, at least for $n$ sufficiently large,

$$
|\mathcal{F}| \leq 2\left(\begin{array}{l}
n-1 \\
k-1
\end{array}\right)-\left(\begin{array}{l}
n-2 \\
k-2
\end{array}\right)+\left(\begin{array}{l}
n-4 \\
k-2
\end{array}\right)
$$

with equality only if $\mathcal{F}$ has the described structure, see Theorem 2.

Now for our full generalisation. Let $G$ be a graph on vertex set $[n]$. (The above example can be considered to be the case where $G$ is a cycle.) For $X \subseteq[n]$ let

$$
N(X)=X \cup\left\{y \in[n]: \exists x \in X \text { such that } x \sim_{G} y\right\} .
$$

We say that a family of sets, $\mathcal{F} \subseteq 2^{[n]}$, is $G$-intersecting if

$$
X, Y \in \mathcal{F} \Rightarrow N(X) \cap Y \neq \emptyset \text {. }
$$

In other words, $\mathcal{F}$ is $G$-intersecting if for all $X, Y \in \mathcal{F}$ there exist $x \in X$ and $y \in Y$ such that $x=y$ or $x \sim_{G} y$. For a fixed graph $G$ how large can a $k$-uniform $G$ intersecting family be? For a graph $G$ on vertex set $[n]$ and $k$ a positive integer let

$$
N(G, k)=\max \left\{|\mathcal{F}|: \mathcal{F} \subseteq\left(\begin{array}{c}
{[n]} \\
k
\end{array}\right) \text { and } \mathcal{F} \text { is } G \text {-intersecting }\right\} .
$$

This quantity can be easily computed in some cases. For example, for the complete graph we clearly have $N\left(K_{n}, k\right)=\left(\begin{array}{l}n \\ k\end{array}\right)$. Let $G=K_{a, n-a}$ be the complete bipartite graph having parts $A, B \subseteq[n]$. A $G$-intersecting family $\mathcal{F}$ can be partitioned into 3 families, $\mathcal{F}=\mathcal{F}_{1} \cup \mathcal{F}_{2} \cup \mathcal{F}_{3}$, where $X \in \mathcal{F}_{1}$ implies $X$ meets both $A$ and $B, \mathcal{F}_{2}$ is an intersecting family of subsets of $A$, and $\mathcal{F}_{3}$ is an intersecting family of subset of $B$. It follows that

$$
N\left(K_{a, n-a}, k\right)=\left(\begin{array}{l}
n \\
k
\end{array}\right)-\left(\begin{array}{l}
a \\
k
\end{array}\right)-\left(\begin{array}{c}
n-a \\
k
\end{array}\right)+\left(\begin{array}{l}
a-1 \\
k-1
\end{array}\right)+\left(\begin{array}{c}
n-a-1 \\
k-1
\end{array}\right) .
$$

As another simple example consider the case of the union $H_{p}$ of a clique on $\{1,2, \ldots, p\}$ and an independent set on $\{p+1, p+2, \ldots, n\}$. Here we can show that

$$
N\left(H_{p}, k\right)=\left\{\begin{aligned}
\left(\begin{array}{c}
n \\
k
\end{array}\right)-\left(\begin{array}{c}
n-p \\
k
\end{array}\right): & k \leq n / 2 \\
\left(\begin{array}{l}
n \\
k
\end{array}\right): & k>n / 2
\end{aligned}\right.
$$

The case $k>n / 2$ is trivial and so assume that $k \leq n / 2$. We use induction on $p$. $H_{1}$ is an independent set and so $\mathcal{F}$ is $H_{1}$-intersecting iff it is intersecting. Thus $|\mathcal{F}| \leq$ $\left(\begin{array}{l}n-1 \\ k-1\end{array}\right)=\left(\begin{array}{l}n \\ k\end{array}\right)-\left(\begin{array}{c}n-1 \\ k\end{array}\right)$. For $p \geq 2$ let $\mathcal{F}=\mathcal{F}_{1} \cup \mathcal{F}_{2}$ where $\mathcal{F}_{1}=\{X \in \mathcal{F}: p \notin X\}$. $\mathcal{F}_{1}$ is 
$H_{p-1}^{\prime}$-intersecting where $H_{p-1}^{\prime}=H_{p}-\{p\}$ and so, by induction, $\left|\mathcal{F}_{1}\right| \leq\left(\begin{array}{c}n-1 \\ k\end{array}\right)-\left(\begin{array}{c}n-p \\ k\end{array}\right)$. Clearly $\left|\mathcal{F}_{2}\right| \leq\left(\begin{array}{c}n-1 \\ k-1\end{array}\right)$ and the result follows. Taking $\mathcal{F}=\{X: X \cap[p] \neq \emptyset\}$ shows the bound is tight.

The extremal $G$-intersecting families are analogous to the extremal intersecting families given by Erdős-Ko-Rado for sparse $G$ and small $k$. To make this statement more precise, we first note that if $K \subseteq[n]$ is a clique in $G$ then

$$
\mathcal{F}(K):=\left\{X \subseteq\left(\begin{array}{c}
{[n]} \\
k
\end{array}\right): X \cap K \neq \emptyset\right\}
$$

is a $G$-intersecting family. It follows that

$$
N(G, k) \geq\left(\begin{array}{l}
n \\
k
\end{array}\right)-\left(\begin{array}{c}
n-\omega \\
k
\end{array}\right)
$$

where $\omega=\omega(G)$ is the clique number of $G$. Note that if $G$ is the empty graph then Erdős-Ko-Rado implies $N(G, k)=\left(\begin{array}{c}n-1 \\ k-1\end{array}\right), N(G, k)$ equals the bound given in (3) and any extremal family is of the form given in (2). However, for most graphs we cannot replace the inequality in (3) with equality. If $K$ is a clique in $G$ and $M_{1}, M_{2}, \ldots, M_{r} \subseteq[n] \backslash K$ satisfy

$$
K \subseteq N\left(M_{i}\right) \text { for } i=1, \ldots, r \quad \text { and } \quad M_{i} \cap N\left(M_{j}\right) \neq \emptyset \text { for } i \neq j
$$

then the collection

$$
\mathcal{F}\left(K ; M_{1}, \ldots, M_{r}\right)=\left\{X \subseteq\left(\begin{array}{c}
{[n]} \\
k
\end{array}\right): X \cap K \neq \emptyset \text { or } M_{i} \subseteq X \text { for some } i\right\}
$$

is $G$-intersecting. Thus, if there exists a maximum clique $K$ and $M \subseteq[n] \backslash K$ such that $|M| \leq k$ and $K \subseteq N(M)$ then the bound given in (3) is not the truth. However, for some graphs we are able to show that any maximum family is of the form given in (5).

Theorem 1. Let $G$ be a graph on vertex set $[n]$ and $k$ a positive integer such that

$$
n \geq \frac{2 e(1+\Delta) \Delta^{\prime}(k-1)^{2} k(k+1)}{\omega}
$$

where $\Delta=\Delta(G)$ is the maximum degree of $G, \omega=\omega(G)$ is the clique number of $G$, and $\Delta^{\prime}=\Delta^{\prime}(G)$ is the maximum size of a second neighborhood in $G$ :

$$
\Delta^{\prime}(G):=\max _{v \in[n]}\left|\left\{i \in[n]: \operatorname{dist}_{G}(i, v) \leq 2\right\}\right| .
$$

If $\mathcal{F}$ is a G-intersecting family containing more than $\left(\begin{array}{c}n \\ k\end{array}\right)-\left(\begin{array}{c}n-\omega \\ k\end{array}\right)$ sets, then there exists a clique $K$ in $G$ and $M_{1}, \ldots, M_{r} \subseteq[n] \backslash K$ such that (4) holds and $\mathcal{F}=$ $\mathcal{F}\left(K ; M_{1}, \ldots, M_{r}\right)$. 
In some sense, Theorem 1 is analogous to the results of Hilton-Milner [6] and HajnalRothschild [7] on intersecting systems which state that an intersecting family that is 'nearly' maximum is 'very similar' in structure to the maximum intersecting families.

Note that Theorem 1 contains information about neither the clique $K$ (e.g. must it be a clique on $\omega(G)$ vertices?) nor the collection of sets $M_{1}, \ldots, M_{r}$. Therefore, when we apply this theorem we must optimize over all possible choices of these parameters. We do this for three classes of graphs.

Corollary 2. Let $G=C_{n}^{p}$ be the $p^{\text {th }}$ power of a cycle on $n$ vertices and $k$ be a positive integer. If

$$
n \geq \frac{2 e(2 p+1)(4 p+1)(k-1)^{2} k(k+1)}{p+1}
$$

then

$$
N(G, k)=\left(\begin{array}{l}
n \\
k
\end{array}\right)-\left(\begin{array}{c}
n-2 p-1 \\
k
\end{array}\right)-p\left(\begin{array}{c}
n-2 p-2 \\
k-1
\end{array}\right) .
$$

Furthermore, a G-intersecting family $\mathcal{F}$ has cardinality $N(G, k)$ if and only if $\mathcal{F}=$ $\mathcal{F}\left(K, M_{1}, \ldots, M_{\left(\begin{array}{c}p+1 \\ 2\end{array}\right)}\right)$ where $K$ is a clique on $p+1$ elements and

$$
\left\{M_{1}, \ldots, M_{\left(\begin{array}{c}
p+1 \\
2
\end{array}\right)}\right\}=\left\{\{i, j\} \in\left(\begin{array}{c}
{[n] \backslash K} \\
2
\end{array}\right): i \varkappa_{G} j \text { and } K \subseteq N(\{i, j\})\right\} \text {. }
$$

Corollary 3. Let $G$ be a d-regular graph on vertex set $[n]$ having no cycle on 5 or fewer vertices. If

$$
\begin{gathered}
k<d, \\
n \geq(k+1)+2 e(k-2) k(d+1)(d-1)^{k} \text { and } \\
n \geq e(d+1)\left(d^{2}+d+1\right)(k-1)^{2} k(k+1)
\end{gathered}
$$

then

$$
N(G, k)=\left(\begin{array}{l}
n \\
k
\end{array}\right)-\left(\begin{array}{c}
n-2 \\
k
\end{array}\right)+\sum_{i=1}^{k-2}\left[\sum_{j=0}^{i}(-1)^{j}\left(\begin{array}{l}
i \\
j
\end{array}\right)\left(\begin{array}{c}
n-j(d-1)-i-2 \\
k-1
\end{array}\right)\right] .
$$

Furthermore, a $G$-intersecting family has cardinality $N(G, k)$ if and only if it has the following form:

$$
\mathcal{F}=\mathcal{G}_{0} \cup \mathcal{G}_{1} \cup \cdots \cup \mathcal{G}_{k-2}
$$

where

$$
\mathcal{G}_{0}=\left\{X \in\left(\begin{array}{c}
{[n]} \\
k
\end{array}\right): X \cap\{x, y\} \neq \emptyset\right\}
$$


and for $1 \leq i \leq k-2$

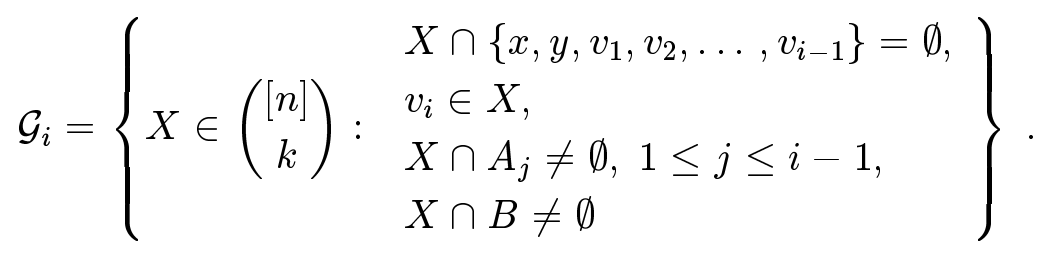

Here

$$
\begin{aligned}
& \{x, y\} \text { is an edge of } G, \text { i.e., a maximum clique, } \\
& N(\{x\}) \backslash\{x, y\}=\left\{v_{1}, v_{2}, \ldots, v_{d-1}\right\} \\
& A_{j}=N\left(\left\{v_{j}\right\}\right) \backslash\left\{v_{j}, x\right\}, 1 \leq j \leq d-1 \\
& B=N(\{y\}) \backslash\{x, y\}
\end{aligned}
$$

Remark 4. Unfortunately, the $p$-cube $Q_{p}$ on $n=2^{p}$ vertices does not fit the framework of Corollary 3, it has cycles of length 4. It is possible to determine the maximal families for small values of $k$, but the complexity of their description soon gets out of hand. For example, if $k \leq 3$ and

$$
n \geq e(p+1)\left(p+1+\left(\begin{array}{c}
p+1 \\
2
\end{array}\right)\right)(k-1)^{2} k(k+1)
$$

then

$$
N\left(Q_{p}, k\right)=\left(\begin{array}{l}
n \\
k
\end{array}\right)-\left(\begin{array}{c}
n-2 \\
k
\end{array}\right)+\left(\begin{array}{l}
n-3 \\
k-1
\end{array}\right)+\left(\begin{array}{l}
n-4 \\
k-1
\end{array}\right)-2\left(\begin{array}{c}
n-p-2 \\
k-1
\end{array}\right) .
$$

While we must rely on the structure of individual graphs to determine if families of the form given in (5) are extremal and to determine the value of $N(G, k)$ these families give, we would expect that for most graphs (not just sparse graphs) and small $k$ the dominant term in $N(G, k)$ is $\left(\begin{array}{l}n \\ k\end{array}\right)-\left(\begin{array}{c}n-\omega \\ k\end{array}\right)$. In order to prove a version of this assertion, we appeal to the language of random graphs. Let $p, 0<p<1$, be a fixed real number. For the random graph $G_{n, p}$ we consider $N\left(G_{n, p}, k\right)$ where $k$ is fixed and $n$ goes to infinity. Since the clique number of $G_{n, p}$ is $\mathbf{w h p}^{1}(2+o(1)) \log _{1 / p} n$ (see [2]) it follows from (3) that

$$
N\left(G_{n, p}, k\right) \geq(2+o(1)) \log _{1 / p} n \cdot\left(\begin{array}{c}
n-(2+o(1)) \log _{1 / p} n \\
k-1
\end{array}\right)
$$

whp. We show that a constant multiple of this lower bound is an upper bound on $N\left(G_{n, p}, k\right)$.

Theorem 5. Let $p, 0<p<1$, be a fixed real number and $k \geq 2$ be a positive integer. Then

$$
\operatorname{Pr}\left(N\left(G_{n, p}, k\right) \geq c_{k, p}\left(\begin{array}{c}
n \\
k-1
\end{array}\right) \lambda_{n}\right)=o(1)
$$

\footnotetext{
${ }^{1} \mathrm{~A}$ sequence of events $\mathcal{E}_{n}$ occurs with high probability, whp, if $\operatorname{Pr}\left(\mathcal{E}_{n}\right)=1-o(1)$.
} 
where $\lambda_{n}=\max \left\{\ln n, \log _{1 / p} n\right\}$ and

$$
c_{k, p}=\frac{8}{(1-p)^{k-1}}\left[\frac{4 k(k-1)}{(1-p)^{(k-1)^{2}}}+1\right] \text {. }
$$

Note that if $k=1$ then $N\left(G_{n, p}, 1\right)$ is given by the clique number of $G_{n, p}$ for any $0<p<1$.

For many graphs $N(G, k)$ is nearly equal to $\left(\begin{array}{l}n \\ k\end{array}\right)$ when $k$ is large.

Theorem 6. Let $\delta=\delta(G)$ be the minimum degree of the graph $G$. Then

$$
k(\delta+1)>c n \ln n \quad \Rightarrow \quad N(G, k) \geq\left(1-n^{1-c}\right)\left(\begin{array}{l}
n \\
k
\end{array}\right)
$$

Theorem 7. Let $\delta$ and $c$ be constants satisfying

$$
r:=c-(1-c)^{\delta+1}>0
$$

If $G$ is a graph on vertex set $[n]$ having minimum degree at least $\delta$ and having maximum degree $\Delta(G)=\Delta<\frac{r}{s} \sqrt{\frac{n}{\log n}}$ and $k \geq$ cn then

$$
N(G, k) \geq\left(1-n^{-s^{2} / 2}\right)\left(\begin{array}{l}
n \\
k
\end{array}\right) \text {. }
$$

Now, it follows from (1) that

$$
N\left(K_{\delta, n-\delta},\lfloor c n\rfloor\right) \sim\left[1-(1-c)^{\delta}\right]\left(\begin{array}{l}
n \\
k
\end{array}\right)
$$

for $\delta, c$ fixed constants and $n \rightarrow \infty$. Hence, it is not the case that $N(G, k)$ is nearly $\left(\begin{array}{l}n \\ k\end{array}\right)$ whenever $k$ is on the order of $n$.

We have seen that for most graphs $N(G, k)$ is determined by extremal families that 'cluster' around cliques when $k$ is small and is nearly $\left(\begin{array}{l}n \\ k\end{array}\right)$ when $k$ is large. As there is a considerable gap between the values of $k$ for which these two types of behavior have been observed, this leaves the obvious question: what happens for $k$ in between? Is there a sharp phase transition between these two types of extrema? Do other types of extrema exist? As it seems likely that the answers to these questions will depend on the graph in question, we hazard a conjecture for only one graph, the cycle.

Conjecture 8. There exists a constant $c$ such that for any fixed $\epsilon>0$

$$
\begin{aligned}
& k \leq(c-\epsilon) n \Rightarrow N\left(C_{n}, k\right)=2\left(\begin{array}{l}
n-1 \\
k-1
\end{array}\right)-\left(\begin{array}{l}
n-2 \\
k-2
\end{array}\right)+\left(\begin{array}{l}
n-4 \\
k-2
\end{array}\right) . \\
& k \geq(c+\epsilon) n \Rightarrow N\left(C_{n}, k\right)=(1-o(1))\left(\begin{array}{l}
n \\
k
\end{array}\right)
\end{aligned}
$$

Note that it follows from Theorem 7 that $N\left(C_{n}, k\right)$ is nearly $\left(\begin{array}{l}n \\ k\end{array}\right)$ for $k>.32 n$.

The remainder of this paper is organized as follows. Theorems 6 and 7 are proven in the next section. In Section 3 we first prove Theorem 1 and then show how Corollaries 2 and 3. Finally, in Section 4, we prove Theorem 5. 


\section{$2 \quad$ Large $\mathrm{k}$}

Both proofs in this section are probabilistic, and for both proofs we restrict our attention to the probability space $\left(\begin{array}{c}{[n]} \\ k\end{array}\right)$ with the uniform measure. Let $X_{n, k}$ denote a random element of this probability space.

Proof of Theorem 6. Let

$$
\mathcal{F}=\left\{X \in\left(\begin{array}{c}
{[n]} \\
k
\end{array}\right): N(X)=[n]\right\} .
$$

Clearly, $\mathcal{F}$ is a $G$-intersecting family. For a fixed vertex $v \in[n]$ we have

$$
\begin{aligned}
\mathrm{P}\left(v \notin N\left(X_{n, k}\right)\right) & =\prod_{i=0}^{\operatorname{deg}_{G}(v)}\left(1-\frac{k}{n-i}\right) \\
& \leq\left(1-\frac{k}{n}\right)^{\delta+1} \\
& <e^{-k(\delta+1) / n} .
\end{aligned}
$$

Therefore, by the first moment method,

$$
\mathrm{P}\left(X_{n, k} \notin \mathcal{F}\right)<n e^{-k(\delta+1) / n} .
$$

Proof of Theorem 7. Let

$$
\mathcal{F}=\left\{X \in\left(\begin{array}{c}
{[n]} \\
k
\end{array}\right):|N(X)|>n-k\right\} .
$$

Clearly, $\mathcal{F}$ is a $G$-intersecting family. For $1 \leq i \leq n$ let $Y_{i}$ be the random variable

$$
Y_{i}(X)=\mathbf{E}\left[\left|\overline{N\left(X_{n, k}\right)}\right|: X_{n, k} \cap[i]=X \cap[i]\right]
$$

(here $\overline{N\left(X_{n, k}\right)}=[n] \backslash N\left(X_{n, k}\right)$ ), and let $Y_{0}$ be the (constant) random variable $Y_{0}=$ $\mathbf{E}\left[\left|\overline{N\left(X_{n, k}\right)}\right|\right] . Y_{0}, \ldots, Y_{n}$ is a martingale. Since

$$
\mathbf{E}\left[\left|\overline{N\left(X_{n, k}\right)}\right|\right] \leq \sum_{i=1}^{n}\left(1-\frac{k}{n}\right)^{\operatorname{deg}_{G}(i)+1} \leq(1-c)^{\delta+1} n
$$

and

$$
\left|Y_{i}-Y_{i-1}\right| \leq \Delta
$$

it follows from Azuma's inequality (see [1, page 85]) that for $\lambda>0$

$$
\mathrm{P}\left(Y_{n}>(1-c)^{\delta+1} n+\lambda \Delta \sqrt{n}\right)<e^{-\lambda^{2} / 2} .
$$

Since $\mathrm{P}\left(X_{n, k} \notin \mathcal{F}\right) \leq \mathrm{P}\left(Y_{n} \geq c n\right)$, the result follows. 


\section{Sparse graphs, small k}

In this section we prove Theorem 1 and Corollaries 2 and 3 . We first prove two general Lemmas from which Theorem 1 follows directly, and then go on to prove the corollaries. Throughout this section we assume $G$ is a graph on vertex set $[n]$ and $k$ is a positive integer satisfying

$$
n \geq \frac{2 e(1+\Delta) \Delta^{\prime}(k-1)^{2} k(k+1)}{\omega}
$$

where $\Delta=\Delta(G)$ is the maximum degree of $G, \omega=\omega(G)$ is the clique number of $G$, and $\Delta^{\prime}=\Delta^{\prime}(G)$ is the maximum size of a second neighborhood in $G$ :

$$
\Delta^{\prime}(G):=\max _{v \in[n]}\left|\left\{i \in[n]: \operatorname{dist}_{G}(i, v) \leq 2\right\}\right| .
$$

Note that the assumptions of Corollaries 2 and 3 imply (11). We further assume $\mathcal{F}$ is a large $G$-intersecting family; in particular, we assume $\mathcal{F}$ is a $G$-intersecting family satisfying

$$
|\mathcal{F}|>\left(\begin{array}{l}
n \\
k
\end{array}\right)-\left(\begin{array}{c}
n-\omega \\
k
\end{array}\right) .
$$

At this point, we must introduce some notation and make some preliminary observations. For $i \in[n]$ let

$$
\mathcal{F}_{i}=\{X \in \mathcal{F}: i \in X\} \text { and } \nu_{i}=\left|\mathcal{F}_{i}\right|
$$

We set $\nu_{\max }$ to be the largest of the $\nu_{i}$ 's. Since, for $X \in \mathcal{F}$ fixed, every set in $\mathcal{F}$ intersects $N(X)$ we have

$$
|\mathcal{F}| \leq|N(X)| \nu_{\max } \leq(\Delta+1) k \nu_{\max }
$$

and so

$$
\nu_{\max }>\frac{1}{k(\Delta+1)}\left[\left(\begin{array}{l}
n \\
k
\end{array}\right)-\left(\begin{array}{c}
n-\omega \\
k
\end{array}\right)\right] .
$$

Finally, we say a vertex $i \in[n]$ is saturated if

$$
\nu_{i}>\frac{1}{k(\Delta+1)}\left[\left(\begin{array}{l}
n \\
k
\end{array}\right)-\left(\begin{array}{c}
n-\omega \\
k
\end{array}\right)\right] .
$$

Note that it follows from (13) that there exists at least one saturated vertex. We will make use of the following inequality:

$$
a, b \geq 2, m>a b \quad \Rightarrow \quad\left(\begin{array}{c}
m-2 \\
b-2
\end{array}\right)<\frac{2 e(b-1)}{m}\left(\begin{array}{c}
m-a \\
b-1
\end{array}\right) .
$$


Proof of (14). Let $a, b, m$ be integers such that $a, b \geq 2$ and $a b<m$.

$$
\begin{aligned}
\frac{\left(\begin{array}{c}
m-2 \\
b-2
\end{array}\right)}{\left(\begin{array}{c}
m-a \\
b-1
\end{array}\right)} & <\frac{b-1}{m-a}\left(\frac{m-b+1}{m-a-b+2}\right)^{b-2} \\
& <\frac{2(b-1)}{m}\left(1+\frac{a-1}{m-a-b+2}\right)^{b-2} \\
& <\frac{2(b-1)}{m} e^{\frac{(a-1)(b-2)}{m-a-b+2}} \\
& <\frac{2 e(b-1)}{m} .
\end{aligned}
$$

Lemma 9. If $v$ is a saturated vertex then

$$
v \in N(X) \quad \forall X \in \mathcal{F}
$$

Proof. Let $\mathcal{F}_{v}^{\prime}=\left\{X \backslash\{v\}: X \in \mathcal{F}_{v}\right\}$. We will call a collection $\mathcal{H} \subseteq \mathcal{F}_{v}^{\prime}$ separated if

$$
\forall X, Y \in \mathcal{H} \quad \operatorname{dist}_{G}(X, Y) \geq 3 .
$$

Let $\mathcal{H}$ be a maximum separated collection in $\mathcal{F}_{v}^{\prime}$. The number of vertices at a distance less than or equal to 2 from an element of $\mathcal{H}$ is at most

$$
M=|\mathcal{H}|(k-1) \Delta^{\prime} .
$$

Using the maximality of $\mathcal{H},(11)$, and (14) we have

$$
\begin{aligned}
\left|\mathcal{F}_{v}\right| & \leq\left(\begin{array}{l}
n-1 \\
k-1
\end{array}\right)-\left(\begin{array}{c}
n-M-1 \\
k-1
\end{array}\right) \\
& \leq M\left(\begin{array}{l}
n-2 \\
k-2
\end{array}\right) \\
& \leq \frac{M 2 e(k-1)}{n}\left(\begin{array}{c}
n-\omega \\
k-1
\end{array}\right) \\
& <\frac{M 2 e(k-1)}{n \omega}\left[\left(\begin{array}{l}
n \\
k
\end{array}\right)-\left(\begin{array}{c}
n-\omega \\
k
\end{array}\right)\right] \\
& <\frac{|\mathcal{H}|}{k(k+1)(\Delta+1)}\left[\left(\begin{array}{l}
n \\
k
\end{array}\right)-\left(\begin{array}{c}
n-\omega \\
k
\end{array}\right)\right] .
\end{aligned}
$$

Since $v$ is a saturated vertex we have

$$
|\mathcal{H}| \geq k+1
$$

Now consider an arbitrary $X \in \mathcal{F}$. By the pigeonhole principle, some vertex of $X$ must be adjacent to at least two sets in $\mathcal{H}$. Since $\mathcal{H}$ is separated, such a vertex can only be an element of $N(\{v\})$. 
Lemma 10. If $u$ and $v$ are saturated vertices then $u \sim_{G} v$.

Proof. Let $u$ and $v$ be saturated vertices and assume for the sake of contradiction that $u \chi_{G} v$. It follows from Lemma 9 that every element of $\mathcal{F}_{u}$ intersects $N(\{v\})$. Therefore, using (14),

$$
\begin{aligned}
\left|\mathcal{F}_{u}\right| & \leq(\Delta+1)\left(\begin{array}{l}
n-2 \\
k-2
\end{array}\right) \\
& \leq \frac{(\Delta+1) 2 e(k-1)}{n}\left(\begin{array}{l}
n-\omega \\
k-1
\end{array}\right) \\
& <\frac{(\Delta+1) 2 e(k-1)}{n \omega}\left[\left(\begin{array}{l}
n \\
k
\end{array}\right)-\left(\begin{array}{c}
n-\omega \\
k
\end{array}\right)\right] \\
& <\frac{1}{\left(1+\Delta+\Delta^{2}\right)(k-1) k(k+1)}\left[\left(\begin{array}{l}
n \\
k
\end{array}\right)-\left(\begin{array}{c}
n-\omega \\
k
\end{array}\right)\right] .
\end{aligned}
$$

It follows from Lemma 10 that the saturated vertices form a clique $K$ in $G$, and it follows from Lemma 9 that any set $X \in \mathcal{F}$ satisfies $K \subseteq N(X)$. Thus, we have proven Theorem 1 (note that we can simply take the collection $\left\{M_{1}, \ldots, M_{r}\right\}$ to be those sets in $\mathcal{F}$ that do not intersect $K$ ).

Proof of Corollary 2. For notational convenience we identify the vertex set $[n]$ of $G=$ $C_{n}^{p}$ with the cyclic group $\mathbf{Z}_{n}$ in the natural way; to be precise,

$$
\alpha \sim_{G} \beta \Leftrightarrow \alpha-\beta \in\{1, \ldots, p, n-p, \ldots, n-1\}
$$

where the difference is taken in $\mathbf{Z}_{n}$. Let $\mathcal{F}$ be a maximum $G$-intersecting family. We may assume that $\mathcal{F}$ is of the form given in (5).

If $|K|=p+1$ then $K=\{\alpha, \alpha+1, \ldots, \alpha+p\}$ for some $\alpha \in \mathbf{Z}_{n}$. Furthermore, for each set $M \in\left\{M_{1}, \ldots, M_{r}\right\}$

$$
\exists \alpha+p+x, \alpha-y \in M \text { such that } x, y \in\{1, \ldots, p\} \text { and } x+y \leq p+1 .
$$

However, if $M_{i}$ and $M_{j}$ satisfy (15) then $M_{i} \cap N\left(M_{j}\right) \neq \emptyset$. So, we can take the family $\left\{M_{1}, \ldots, M_{r}\right\}$ to be the collection of all 2 element sets that satisfy (15), and it suffices to show $|K|=p+1$.

Assume for the sake of contradiction that there exists $\alpha \in K$ such that $\alpha+1 \notin K$ and $K \cap\{\alpha+2, \ldots, \alpha+p\} \neq \emptyset$. Since $\mathcal{F}$ is maximum and $\alpha+1$ is not saturated, the set $\mathcal{F}^{\prime}:=\{X \in \mathcal{F}: \alpha+1 \notin N(X)\}$ is nonempty. Therefore,

$$
\left|\mathcal{F}_{\alpha+1}\right| \leq k(2 p+1)\left(\begin{array}{l}
n-2 \\
k-2
\end{array}\right) .
$$


Since an arbitrary element of $\mathcal{F}^{\prime}$ must contain $\alpha-p$ and some element of $\{\alpha+p+$ $2, \ldots, \alpha+2 p\}$ we have

$$
\left|\mathcal{F}^{\prime}\right| \leq(p-1)\left(\begin{array}{l}
n-2 \\
k-2
\end{array}\right) .
$$

It follows from (14) and (6) that

$$
\left|\mathcal{F}_{\alpha+1}\right|+\left|\mathcal{F}^{\prime}\right| \leq[k(2 p+1)+p-1]\left(\begin{array}{l}
n-2 \\
k-2
\end{array}\right)<\left(\begin{array}{l}
n-1 \\
k-1
\end{array}\right) .
$$

Since the family

$$
\left(\mathcal{F} \backslash \mathcal{F}_{\alpha+1} \backslash \mathcal{F}^{\prime}\right) \cup\left\{X \in\left(\begin{array}{c}
{[n]} \\
k-1
\end{array}\right): \alpha+1 \in X\right\}
$$

is $G$-intersecting, this is a contradiction.

Thus, we may assume $K=\{\alpha, \alpha+1, \ldots, \alpha+l\}$ for some $\alpha \in \mathbf{Z}_{n}$ and $l \leq p$. Assume for the sake of contradiction that $l<p$. Let $\mathcal{F}^{\prime \prime}=\{X \in \mathcal{F}: \alpha-1 \notin N(X)\}$. Since an arbitrary element of $\mathcal{F}^{\prime \prime}$ covers $\alpha$ without covering $\alpha-1$, we have $\mathcal{F}^{\prime \prime} \subseteq \mathcal{F}_{\alpha+p}$. Since neither $\alpha-1$ nor $\alpha+p$ is saturated we have

$$
\left|\mathcal{F}_{\alpha-1}\right|+\left|\mathcal{F}_{\alpha+p}\right| \leq \frac{2}{k(2 p+1)}\left[\left(\begin{array}{l}
n \\
k
\end{array}\right)-\left(\begin{array}{c}
n-p-1 \\
k
\end{array}\right)\right]<\frac{2}{k}\left(\begin{array}{l}
n-1 \\
k-1
\end{array}\right) .
$$

This yields a contradiction because the family

$$
\left(\mathcal{F} \backslash \mathcal{F}_{\alpha-1} \backslash \mathcal{F}_{\alpha+p}\right) \cup\left\{X \in\left(\begin{array}{c}
{[n]} \\
k
\end{array}\right): \alpha-1 \in X\right\}
$$

is $G$-intersecting and is larger than $\mathcal{F}$.

To establish (7) we assume that $K=\{1,2, \ldots, p+1\}$. Then we can take

$$
\mathcal{F}=\left(\begin{array}{c}
{[n]} \\
k
\end{array}\right) \backslash\left(\bigcup_{i=0}^{p} \mathcal{G}_{i}\right)
$$

where

$$
\mathcal{G}_{0}=\left\{X \in\left(\begin{array}{c}
{[n]} \\
k
\end{array}\right): X \cap(K \cup\{n-p+1, \ldots, n\})=\emptyset\right\}
$$

and

$$
\mathcal{G}_{i}=\left\{X \in\left(\begin{array}{c}
{[n]} \\
k
\end{array}\right): n-i+1 \in X, X \cap\{n-i+2, \ldots, 2 p+3-i\}=\emptyset\right\} .
$$

Equation (7) follows immediately.

Proof of Corollary 3. Assume $\mathcal{F}$ is a maximum $G$-intersecting family. We may assume $\mathcal{F}$ is of the form given in (5). Let $\mathcal{M}$ be the collection $\left\{M_{1}, \ldots, M_{r}\right\}$ defined in (5). 
We first note that $|K|=2$ : If $x$ is the unique saturated vertex then

$$
\begin{aligned}
|\mathcal{F}| & \leq\left|\mathcal{F}_{x}\right|+\sum_{v: v \sim x}\left|\mathcal{F}_{v}\right| \\
& <\left(\begin{array}{l}
n-1 \\
k-1
\end{array}\right)+\frac{d}{k(d+1)}\left[\left(\begin{array}{l}
n \\
k
\end{array}\right)-\left(\begin{array}{c}
n-2 \\
k
\end{array}\right)\right] \\
& <\left(\begin{array}{l}
n \\
k
\end{array}\right)-\left(\begin{array}{c}
n-2 \\
k
\end{array}\right)
\end{aligned}
$$

which is a contradiction.

Now, let $K=\{x, y\}$,

$$
A=N(\{x\}) \backslash\{x, y\} \text { and } B=N(\{y\}) \backslash\{x, y\} .
$$

For $v \in A \cup B$ let

$$
A_{v}=N(\{v\}) \backslash\{x, y, v\}
$$

Note that since $G$ contains no small cycle, these sets are pairwise disjoint. Furthermore, the only edges between these sets are those connecting $v \in A \cup B$ with $A_{v}$ and possibly edges connecting $A_{v}$ and $A_{u}$ where $u \in A$ and $v \in B$.

It remains to determine the size and structure of

$$
\mathcal{F}_{0}:=\{X \in \mathcal{F}: X \cap K=\emptyset\}
$$

It follows from Theorem 1 that all elements of $\mathcal{F}_{0}$ intersect both $A$ and $B$ (it follows immediately that $\mathcal{F}_{0}$ is empty for $k=1$; we henceforth assume $k \geq 2$ ). We proceed in stages. In the first stage we show that there exists a vertex $v_{1} \in A \cup B$ (we assume without loss of generality that $v_{1} \in A$ ) such that

$$
\mathcal{M}_{1}:=\left\{\left\{v_{1}, b\right\}: b \in B\right\}
$$

is a subset of $\mathcal{M}$ and that all sets in

$$
\mathcal{F}_{1}:=\left\{X \in \mathcal{F}_{0}: \nexists Y \in \mathcal{M}_{1} \text { such that } Y \subseteq X\right\}
$$

must intersect $A_{1}:=A_{v_{1}}$. Since $\mathcal{F}_{1} \subseteq \mathcal{F}_{0}$ all sets in $\mathcal{F}_{1}$ must intersect $A_{1}, A \backslash\left\{v_{1}\right\}$ and $B$ (note that any set in $\mathcal{F}_{0}$ that contains $v_{1}$ must contain some set in $\mathcal{M}_{1}$ ). In stage $2 \leq i \leq k-1$ we show that there exists a vertex $v_{i} \in A \backslash\left\{v_{1}, \ldots, v_{i-1}\right\}$ such that

$$
\mathcal{M}_{i}:=\left\{Y \in\left(\begin{array}{c}
{[n]} \\
i+1
\end{array}\right): v_{i} \in Y \text { and } Y \text { intersects } B, A_{1}, \ldots, A_{i-1}\right\}
$$

is a subset of $\mathcal{M}$ and that all sets in

$$
\mathcal{F}_{i}:=\left\{X \in \mathcal{F}_{i-1}: \nexists Y \in \mathcal{M}_{i} \text { such that } Y \subseteq X\right\}
$$


must intersect $A_{i}$. Thus, all sets in $\mathcal{F}_{i}$ must intersect $A \backslash\left\{v_{1}, \ldots, v_{i}\right\}, B, A_{1}, \ldots, A_{i}$. We have a complete characterization of $\mathcal{F}$ after the $(k-1)$ st stage because $\mathcal{F}_{k-1}$ is empty: an element of $\mathcal{F}_{k-1}$ has $k$ elements and intersects $A \backslash\left\{v_{1}, \ldots, v_{k-1}\right\}, B, A_{1}$, $\ldots, A_{k-1}$.

We begin with the first stage. As noted above, every element of $\mathcal{F}_{0}$ must intersect both $A$ and $B$. For $(a, b) \in A \times B$ let

$$
\mathcal{F}_{a, b}=\left\{X \in \mathcal{F}_{0}:\{a, b\} \subseteq X\right\} .
$$

Let $\mathcal{P}$ be the set of pairs $(a, b)$ for which $\mathcal{F}_{a, b}$ is nonempty. We say that the pair $(a, b) \in \mathcal{P}$ covers $\left(a^{\prime}, b^{\prime}\right) \in \mathcal{P}$ if

$$
X \in \mathcal{F}_{a, b} \Rightarrow N(X) \cap\left\{a^{\prime}, b^{\prime}\right\} \neq \emptyset .
$$

Now, if $(a, b) \in \mathcal{P}$ does not cover $\left(a^{\prime}, b^{\prime}\right)$ then there exists $X \in \mathcal{F}_{a, b}$ such that $N(X) \cap$ $\left\{a^{\prime}, b^{\prime}\right\}=\emptyset$, and

$$
\left|\mathcal{F}_{a^{\prime}, b^{\prime}}\right| \leq|N(X)|\left(\begin{array}{l}
n-5 \\
k-3
\end{array}\right) \leq(d+1) k\left(\begin{array}{l}
n-5 \\
k-3
\end{array}\right) .
$$

On the other hand, if $(a, b)$ covers $\left(a^{\prime}, b^{\prime}\right)$ and $\{a, b\} \cap\left\{a^{\prime}, b^{\prime}\right\}=\emptyset$ then every set in $\mathcal{F}_{a, b}$ contains some element of $A_{a^{\prime}} \cup A_{b^{\prime}}$, and

$$
\left|\mathcal{F}_{a, b}\right| \leq 2(d-1)\left(\begin{array}{l}
n-5 \\
k-3
\end{array}\right)
$$

We call a pair $(a, b) \in \mathcal{P}$ good if every pair in $\mathcal{P}$ covers $(a, b)$ and

$$
(a, b) \text { covers }\left(a^{\prime}, b^{\prime}\right) \Rightarrow\{a, b\} \cap\left\{a^{\prime}, b^{\prime}\right\} \neq \emptyset .
$$

Note that if $(a, b)$ is good then we may assume that $\mathcal{F}$ contains all sets that contain $\{a, b\}$. A pair $(a, b) \in \mathcal{P}$ that is not good will be called $b a d$. It follows from (16) and (17) that

$$
\left|\mathcal{F}_{a, b}\right| \leq(d+1) k\left(\begin{array}{l}
n-5 \\
k-3
\end{array}\right)
$$

for any bad pair $(a, b)$. Thus, the number of sets in $\mathcal{F}$ containing at least one bad pair is at most

$$
d^{2}(d+1) k\left(\begin{array}{l}
n-5 \\
k-3
\end{array}\right)
$$

By (8) and (14) this quantity is less than $\left(\begin{array}{c}n-2 d \\ k-2\end{array}\right)$ which is a lower bound on the number of sets containing some fixed good pair (and no other good pair). Thus, $\mathcal{F}$ has the maximum possible number of good pairs. 
How many good pairs could $\mathcal{F}$ have? Note that if $(a, b)$ and $\left(a^{\prime}, b^{\prime}\right)$ are good pairs then $\{a, b\} \cap\left\{a^{\prime}, b^{\prime}\right\} \neq \emptyset$. Clearly, we optimize the number of good pairs by taking all pairs that contain some fixed vertex. Therefore, there exists a vertex $v_{1}$ such that the collection

$$
\mathcal{M}_{1}=\left\{\left\{v_{1}, b\right\}: b \in B\right\}
$$

is a subset of $\mathcal{M}$. Furthermore, by the definition of a good pair and the condition $k<d$, any set in $\mathcal{F}_{1}$ (i.e. any set in $\mathcal{F}_{0}$ that does not contain some set in $\mathcal{M}_{1}$ ) must intersect $A_{1}$. We have completed the first stage.

We now proceed by induction. Suppose stage $l$ for $1 \leq l \leq k-2$ has been completed as described above. Each set in $\mathcal{F}_{l}$, the collection of sets in $\mathcal{F}$ that have not yet been characterized (i.e. sets in $\mathcal{F}$ that neither intersect $K$ nor contain a set in $\left.\mathcal{M}_{1} \cup \cdots \cup \mathcal{M}_{l}\right)$, intersects $A \backslash\left\{v_{1}, \ldots, v_{l}\right\}, B, A_{1}, \ldots$, and $A_{l}$. We proceed as above: For

$$
\mu=\left(a, b, x_{1}, \ldots, x_{l}\right) \in\left(A \backslash\left\{v_{1}, \ldots, v_{l}\right\}\right) \times B \times A_{1} \times \cdots \times A_{l}
$$

we define

$$
\mathcal{F}_{\mu}=\left\{X \in \mathcal{F}_{l}:\left\{a, b, x_{1}, \ldots, x_{l}\right\} \subseteq X\right\}
$$

and set

$$
\mathcal{P}_{l}=\left\{\mu \in A \times B \times A_{1} \times \cdots \times A_{l}: \mathcal{F}_{\mu} \neq \emptyset\right\} .
$$

We say that $\mu \in \mathcal{P}_{l}$ covers $\mu^{\prime}=\left(a^{\prime}, b^{\prime}, x_{1}^{\prime}, \ldots, x_{l}^{\prime}\right) \in \mathcal{P}_{l}$ if

$$
X \in \mathcal{F}_{\mu} \Rightarrow N(X) \cap\left\{a^{\prime}, b^{\prime}, x_{1}^{\prime}, \ldots, x_{l}^{\prime}\right\} \neq \emptyset
$$

and that $\mu=\left(a, b, x_{1}, \ldots, x_{l}\right) \in \mathcal{P}_{l}$ is good if every $\mu^{\prime} \in \mathcal{P}_{l}$ covers $\mu$ and

$$
\mu \text { covers }\left(a^{\prime}, b^{\prime}, x_{1}^{\prime}, \ldots x_{l}^{\prime}\right) \Rightarrow\left\{a, b, x_{1}, \ldots, x_{l}\right\} \cap\left\{a^{\prime}, b^{\prime}, x_{1}^{\prime}, \ldots x_{l}^{\prime}\right\} \neq \emptyset .
$$

Following the argument above, we conclude that $\mathcal{F}_{l}$ must have the maximum number of good vectors. This maximum is achieved by taking the set of good vectors to be all vectors that contain some fixed vertex. As the number of good vectors is then the product of the cardinalities of the sets that do not contain this fixed vertex, we must take the fixed vertex to be in the smallest of the sets: $A \backslash\left\{v_{1}, \ldots, v_{l}\right\}$.

Now put $\mathcal{G}_{i}=\mathcal{F}_{i} \backslash \mathcal{F}_{i+1}$ for $0 \leq i \leq k-2$ to obtain the collection described in the statement of the theorem.

Then to obtain (10) we see that

$$
\left|\mathcal{G}_{0}\right|=\left(\begin{array}{l}
n \\
k
\end{array}\right)-\left(\begin{array}{c}
n-2 \\
k
\end{array}\right)
$$


and

$$
\left|\mathcal{G}_{i}\right|=\sum_{j=0}^{i}(-1)^{j}\left(\begin{array}{l}
i \\
j
\end{array}\right)\left(\begin{array}{c}
n-j(d-1)-i-2 \\
k-1
\end{array}\right)
$$

for $1 \leq i \leq k-2$.

\section{Random graphs}

We begin with definitions and notation. For finite sets $A, B$, not necessarily disjoint, let $K(A, B)$ be the graph on vertex set $A \cup B$ having edge set

$$
\{\{a, b\}: a \in A, b \in B\} .
$$

If $A, B \subseteq V(G)$ are disjoint vertex sets in a graph $G$ then we set

$$
e_{G}(A, B)=e(A, B)=|\{\{x, y\} \in E(G): x \in A, y \in B\}| .
$$

For an arbitrary hypergraph $\mathcal{H}$ we set $\nu(\mathcal{H})$ equal to the size of the largest matching in $\mathcal{H}$ (i.e. collection of pairwise disjoint edges) and $\tau(\mathcal{H})$ equal to the size of the smallest vertex cover in $\mathcal{H}$ (i.e. collection of vertices that meets all edges). Clearly,

$$
\mathcal{H} \quad k \text {-uniform } \Rightarrow \tau(\mathcal{H}) \leq k \nu(\mathcal{H})
$$

Proof. To prove Theorem 5 we fix $0<p<1$ and a positive integer $k \geq 2$.

Let $G=G_{n, p}$, and let $\mathcal{F} \subseteq\left(\begin{array}{c}{[n]} \\ k\end{array}\right)$ be a $G$-intersecting family. For $X \subseteq[n]$ let

$$
\mathcal{F}_{X}=\{Y \subseteq[n] \backslash X: Y \cup X \in \mathcal{F}\} \quad \text { and } \quad \nu_{X}=\left|\mathcal{F}_{X}\right|
$$

In other words, $\nu_{X}$ is the number of sets in $\mathcal{F}$ that contain $X$. If $|X|=k-1$ we will think of $\mathcal{F}_{X}$ as a set (rather than a collection of one element sets). We say that $X \in\left(\begin{array}{c}{[n]} \\ k-1\end{array}\right)$ is saturated if

$$
\nu_{X} \geq 8 k(1-p)^{-k+1} \lambda_{n}
$$

(note that this definition of saturated differs from that of Section 3). We form the $(k-1)$-uniform hypergraph

$$
\mathcal{H}=\mathcal{H}_{\mathcal{F}}=\left\{X \in\left(\begin{array}{c}
{[n]} \\
k-1
\end{array}\right): X \text { is saturated }\right\} .
$$

We prove Theorem 5 by showing that for an arbitrary $G$-intersecting family $\mathcal{F}$, the hypergraph $\mathcal{H}_{\mathcal{F}}$ is not too 'spread-out.' One measure of the degree to which a hypergraph is 'spread-out' is the vertex cover number. We first observe that if $\tau(\mathcal{H})$ is small then we have the desired bound on $\mathcal{F}$ : If there exists a vertex cover $A$ of $\mathcal{H}$ such that

$$
|A|<32 k(k-1)(1-p)^{k-k^{2}} \lambda_{n}
$$


then

$$
\begin{aligned}
|\mathcal{F}| & =\frac{1}{k}\left[\sum_{X \text { saturated }} \nu_{X}+\sum_{X \text { not saturated }} \nu_{X}\right] \\
& \leq \frac{1}{k}\left[k \sum_{v \in A} \nu_{\{v\}}+\sum_{X \text { not saturated }} \nu_{X}\right] \\
& \leq|A|\left(\begin{array}{c}
n \\
k-1
\end{array}\right)+\frac{1}{k}\left(\begin{array}{c}
n \\
k-1
\end{array}\right) \cdot 8 k(1-p)^{-k+1} \lambda_{n} \\
& <\left[32 k(k-1)(1-p)^{k-k^{2}}+8(1-p)^{-k+1}\right]\left(\begin{array}{c}
n \\
k-1
\end{array}\right) \lambda_{n} .
\end{aligned}
$$

In order to prove Theorem 5 , we let $\mathcal{F}$ to be a $G$-intersecting family such that

$$
|\mathcal{F}| \geq\left[32 k(k-1)(1-p)^{k-k^{2}}+8(1-p)^{-k+1}\right]\left(\begin{array}{c}
n \\
k-1
\end{array}\right) \lambda_{n} .
$$

We show that such a family $\mathcal{F}$ exists only with probability $o(1)$. It follows from (19) that $\tau(\mathcal{H}) \geq 32 k(k-1)(1-p)^{k-k^{2}} \lambda_{n}$, and by (18) there exists a matching $X_{1}, \ldots, X_{r}$ in $\mathcal{H} \subseteq\left(\begin{array}{c}{[n]} \\ k-1\end{array}\right)$ where

$$
r=\left\lceil 32 k(1-p)^{k-k^{2}} \cdot \lambda_{n}\right\rceil
$$

We show that such a matching exists only with probability $o(1)$.

Why can there not exist a large collection of disjoint saturated sets with high probability? We begin with an observation about pairs of sets in the matching. Consider disjoint $X_{i} \neq X_{j}$ for which $e\left(X_{i}, X_{j}\right)=0$. For

$$
x \in \mathcal{F}_{X_{i}} \quad \text { and } \quad y \in \mathcal{F}_{X_{j}}
$$

it follows from the $G$-intersecting property that one of the following holds:

$$
x \in N\left(X_{j}\right), \quad y \in N\left(X_{i}\right) \quad \text { or } \quad x \sim_{G} y .
$$

In more global terms, if $A=\mathcal{F}_{X_{i}} \backslash N\left(X_{j}\right)$ and $B=\mathcal{F}_{X_{j}} \backslash N\left(X_{i}\right)$ then

$$
K(A, B) \subseteq G
$$

However, if $A$ and $B$ are large then whp such subgraphs do not appear in the random graph $G_{n, p}$.

Lemma 11. If $\mathcal{Q}$ is the event that there exists $A, B \subseteq[n]$ such that

$$
|A|,|B|>2 \log _{1 / p} n \text { and } K(A, B) \subseteq E(G),
$$

then $\operatorname{Pr}(\mathcal{Q})=o(1)$. 
The proof of Lemma 11 is deferred to the end of this section. It follows from Lemma 11 and (20) that we can assume that for $i \neq j$

$$
e\left(X_{i}, X_{j}\right)=0 \Rightarrow \min \left\{\left|\mathcal{F}_{X_{i}} \backslash N\left(X_{j}\right)\right|,\left|\mathcal{F}_{X_{j}} \backslash N\left(X_{i}\right)\right|\right\} \leq 2 \log _{1 / p} n .
$$

We shall see, using (21), that there exists a set $X_{a}$ in the matching for which many of the sets $N\left(X_{j}\right)$ have a large intersection with $\mathcal{F}_{X_{a}}$. We finish the proof by showing that whp neighborhood intersections of this kind do not occur in the random graph $G_{n, p}$.

In order to establish the existence of the set $X_{a}$ mentioned above, we form a digraph $D$ on vertex set $[r]$. We connect $i$ to $j$ with an arc if

$$
e\left(X_{i}, X_{j}\right)=0 \quad \text { and } \quad\left|\mathcal{F}_{X_{i}} \backslash N\left(X_{j}\right)\right|<\left|\mathcal{F}_{X_{j}} \backslash N\left(X_{i}\right)\right|
$$

It follows from (21) that

$$
(i, j) \in D \Rightarrow\left|\mathcal{F}_{X_{i}} \backslash N\left(X_{j}\right)\right| \leq 2 \log _{1 / p} n .
$$

Since $G$ is random, the following lemma shows that $D$ has whp many arcs.

Lemma 12. If $\mathcal{R}$ is the event that there exist disjoint sets $Y_{1}, \ldots, Y_{r} \in\left(\begin{array}{c}{[n]} \\ k-1\end{array}\right)$ such that

$$
\left|\left\{\{i, j\} \in\left(\begin{array}{c}
{[r]} \\
2
\end{array}\right): e\left(Y_{i}, Y_{j}\right)=0\right\}\right| \leq \frac{1}{2}\left(\begin{array}{c}
r \\
2
\end{array}\right)(1-p)^{(k-1)^{2}} .
$$

then $\operatorname{Pr}(\mathcal{R})=o(1)$.

The proof of Lemma 12 is deferred to the end of this section. It follows from Lemma 12 that there exists $a \in[r]$ having out degree in $D$ at least

$$
s:=\left\lceil\frac{1}{r}\left(\frac{1}{2}\left(\begin{array}{l}
r \\
2
\end{array}\right)(1-p)^{(k-1)^{2}}\right)\right\rceil \geq 8 k(1-p)^{-k+1} \lambda_{n}-\frac{1}{4}(1-p)^{(k-1)^{2}} .
$$

It follows from (22) and the fact that $X_{a}$ is saturated that the set $S:=\mathcal{F}_{X_{a}}$ and the collection of disjoint $(k-1)$-sets

$$
\left\{Y_{1}, \ldots, Y_{s}\right\}:=\left\{X_{i}:(a, i) \in D\right\}
$$

satisfy

(1) $|S| \geq s$, and

(2) $\left|N\left(Y_{i}\right) \cap S\right| \geq s-2 \log _{1 / p} n \quad$ for $i=1, \ldots, s$.

We complete the proof by showing that whp such a collection of sets does not appear in $G$. 
Lemma 13. If $\mathcal{S}$ is the event that there exist $S \subseteq[n]$ and a collection of disjoint sets $Y_{1}, Y_{2}, \ldots, Y_{s} \in\left({ }_{k-1}^{[n]}\right)$ satisfying $(1)$ and $(2)$ then $\operatorname{Pr}(\mathcal{S})=o(1)$.

The proof of Lemma 13 is deferred to the end of this section.

Proof of Lemma 11. Let $q=2 \log _{1 / p} n$, and for $j=1, \ldots, q$ let $\mathcal{Q}_{j}$ be the event that there exists $A, B \subseteq[n]$ such that

$$
|A|,|B|=q,|A \cap B|=j \text { and } K(A, B) \subseteq E(G) .
$$

Consider a fixed $j$ and set $l=q-j$. An application of the first moment method yields

$$
\begin{aligned}
\operatorname{Pr}\left(\mathcal{Q}_{j}\right) & \leq\left(\begin{array}{c}
n \\
2 l+j
\end{array}\right)\left(\begin{array}{c}
2 l+j \\
j
\end{array}\right) \cdot p^{l^{2}+2 l j+\left(\begin{array}{c}
j \\
2
\end{array}\right)} \\
& \leq \frac{n^{2 l+j}}{(2 l+j) !} \frac{(2 l+j) !}{(2 l) ! j !} \cdot p^{q^{2}-j^{2} / 2-j / 2} \\
& =\left(n^{q} p^{q^{2}}\right) \cdot n^{l} p^{-j^{2} / 2} \cdot p^{-j / 2} \cdot \frac{1}{(2 l) ! j !} \\
& \leq p^{-j} \cdot \frac{1}{(2 l) ! j !} \\
& =o\left(\frac{1}{\log n}\right) .
\end{aligned}
$$

Proof of Lemma 12. For disjoint sets $Y_{1}, \ldots, Y_{r}$ the random variable

$$
E:=\left|\left\{\{i, j\} \in\left(\begin{array}{c}
{[r]} \\
2
\end{array}\right): e\left(Y_{i}, Y_{j}\right)=0\right\}\right|
$$

is distributed as $\mathrm{B}\left(\left(\begin{array}{l}r \\ 2\end{array}\right),(1-p)^{(k-1)^{2}}\right)$. The Chernoff bound then implies that

$$
\operatorname{Pr}\left[E \leq \frac{1}{2}\left(\begin{array}{l}
r \\
2
\end{array}\right) \cdot(1-p)^{(k-1)^{2}}\right]<\exp \left\{-\frac{r(r-1)}{16} \cdot(1-p)^{(k-1)^{2}}\right\} .
$$

Therefore,

$$
\begin{aligned}
\operatorname{Pr}(\mathcal{R}) & \leq\left(\begin{array}{c}
n \\
k-1
\end{array}\right)^{r} \exp \left\{-\frac{r(r-1)}{16} \cdot(1-p)^{(k-1)^{2}}\right\} \\
& \leq n^{r(k-1)} \exp \left\{-2 r k(1-p)^{-k+1} \lambda_{n}+\frac{r}{16}(1-p)^{(k-1)^{2}}\right\} \\
& \leq n^{r(k-1)} \exp \left\{-2 r k(1-p)^{-k+1} \lambda_{n}+2 k(1-p)^{-k+1} \lambda_{n}+1\right\} \\
& \leq O\left(n^{r(k-1)-(2 r k-2 k)(1-p)^{-k+1}}\right) \\
& =o(1) .
\end{aligned}
$$


Proof of Lemma 13. Let $\hat{s}=s-k+1$. Consider $A, B \subseteq[n]$ such that $|A|=s$ and $|B|=k-1$. The random variable

$$
M_{A, B}=|\{x \in A \backslash B: e(\{x\}, B)=0\}|
$$

is stochastically dominated by $\mathrm{B}\left(\hat{s},(1-p)^{k-1}\right)$. It follows from the Chernoff bound that

$$
\begin{aligned}
\operatorname{Pr}\left(M_{A, B} \leq 2 \log _{1 / p} n\right) & \leq \operatorname{Pr}\left[\mathrm{B}\left(\hat{s},(1-p)^{k-1}\right) \leq \frac{1}{2} \cdot \hat{s}(1-p)^{k-1}\right] \\
& \leq \exp \left\{-\frac{1}{8} \cdot \hat{s}(1-p)^{k-1}\right\}
\end{aligned}
$$

Therefore, the probability that fixed $S, Y_{1}, \ldots, Y_{s}$ satisfy (1) and (2) is at most

$$
\exp \left\{-\frac{1}{8} \cdot \hat{s} s(1-p)^{k-1}\right\} \text {. }
$$

Thus,

$$
\begin{aligned}
\operatorname{Pr}(\mathcal{S}) & \leq\left(\begin{array}{c}
n \\
s
\end{array}\right)\left(\begin{array}{c}
n \\
k-1
\end{array}\right)^{s} \exp \left\{-\frac{1}{8} \cdot \hat{s} s(1-p)^{k-1}\right\} \\
& <n^{s+(k-1) s} \exp \left\{-\frac{1}{8} \cdot \hat{s} s(1-p)^{k-1}\right\}\left(\frac{1}{s !}\right) \\
& \leq \exp \left\{s k \lambda_{n}-\frac{1}{8} \cdot \hat{s} s(1-p)^{k-1}\right\}\left(\frac{1}{s !}\right) \\
& \leq \exp \left\{\frac{s}{8}\left(\frac{(1-p)^{k^{2}-k}}{4}+(k-1)(1-p)^{k-1}\right)\right\}\left(\frac{1}{s !}\right) \\
& =o(1) .
\end{aligned}
$$

\section{References}

[1] N. Alon, J. H. Spencer, The probabilistic method, John Wiley and Sons, 1991.

[2] B. Bollobás, Random graphs, Academic Press, 1985.

[3] B. Bollobás, Combinatorics: set systems, hypergraphs, families of vectors and combinatorial probability, Cambridge University Press, 1986.

[4] M. Deza, P. Frankl, Erdös-Ko-Rado theorem - 22 years later, SIAM J. Alg. Disc. Meth. 4 (1983), 419-431.

[5] P. Erdős, C. Ko, R. Rado, Intersection theorems for systems of finite sets, Quart.

J. Math. Oxford Ser. 212 (1961), 313-320. 
[6] A. J. W. Hilton, E. C. Milner, Some intersection theorems for systems of finite sets, Quart. J. Math. Oxford Ser. 218 (1967), 369-384.

[7] A. Hajnal, B. Rothschild, A generalization of the Erdös-Ko-Rado theorem on finite set systems, JCTA 51 (1989), 24-42.

This research was sponsored in part by National Science Foundation (NSF) grant no. CCR-0122581. 